\title{
Thermal conduction mechanisms in boron nitride nanotubes: Few-shell versus all-shell conduction
}

\author{
Ivana Savić, ${ }^{1, *}$ Derek A. Stewart, ${ }^{2}$ and Natalio Mingo ${ }^{1,3, \dagger}$ \\ ${ }^{1}$ LITEN, CEA-Grenoble, 17 rue des Martyrs, 38054 Grenoble, France \\ ${ }^{2}$ Cornell Nanoscale Facility, Cornell University, Ithaca, New York 14853, USA \\ ${ }^{3}$ University of California-Santa Cruz, Santa Cruz, California 95064, USA
}

(Received 30 October 2008; revised manuscript received 24 November 2008; published 29 December 2008)

\begin{abstract}
It has been argued that the experimentally observed limitation of heat transport through boron nitride nanotubes is due to intershell scattering rather than to inefficient heat transfer to inner shells. Using an atomistic Green's function calculation, we present evidence that on the contrary, intershell or any other type of scattering along the nanotubes is not the main limiting mechanism, and heat conduction restricted to a few layers is responsible for the low thermal conductivities experimentally measured. Our results also indicate that anharmonic scattering in boron nitride is relatively weak, which might lead to considerably larger thermal conductivity for well-contacted nanotubes than previously reported.
\end{abstract}

DOI: 10.1103/PhysRevB.78.235434

PACS number(s): 68.65.-k, 63.22. $-\mathrm{m}, 65.80 .+\mathrm{n}, 66.70 .-\mathrm{f}$

\section{INTRODUCTION}

Recent progress in nanoscale thermal conductivity measurements ${ }^{1}$ has enabled the study of carbon ${ }^{2}$ and boron nitride nanotube ${ }^{3-6}$ thermal transport. ${ }^{7-15}$ This in turn raised questions regarding dominant heat conduction mechanisms in these low-dimensional systems. In particular, the issue of heat confinement in the outermost shells of multiwalled nanotubes and the influence of intershell and anharmonic scattering remain without satisfactory answers. A previous study $^{13}$ argued against the possibility of inefficient heat transfer from the outer to the inner shells and suggested the dominant contribution was due to intershell scattering. The latter explanation implies that comparable heat flows are being carried by each of the shells; whereas the former one implies a very inhomogeneous distribution of flow among shells, with some carrying all the heat and others contributing in a negligible way. Distinguishing experimentally between these two possibilities is extremely hard: one might try to plot thermal conductance versus nanotube diameter, in order to see whether it is proportional to the perimeter or to the cross section. But this does not exclude the possibility that the contact quality may be different depending on the diameter. Thus, a few-shell conduction cannot be discarded based on this argument alone.

There is however a way to clearly ascertain whether conduction is of the homogeneously distributed all-shell type or of the inhomogeneous few-shell type. The idea is to controllably include a competing scattering mechanism along the nanotube (NT), the magnitude of which is theoretically understood, and to see how this affects conductivity. The obvious competing mechanism that one can control is isotope scattering, ${ }^{13}$ which has been theoretically studied in detail for carbon NTs (CNTs). ${ }^{16-18}$ Isotopes do not alter any of the chemical or structural characteristics of the nanotubes, so it is possible to compare specimens with in principle exactly the same structure and contact quality. By looking at the difference between the isotopically pure $\left(100 \%{ }^{11} \mathrm{~B}\right)$ and impure $\left(80 \%{ }^{11} \mathrm{~B}\right.$ and $\left.20 \%{ }^{10} \mathrm{~B}\right)$ conductivities for boron nitride NTs (BNNTs), one can determine whether conduction is of the few-shell or all-shell type. As we show in this paper, the effect of isotopes will be much greater for heat flow carried by a few nanotube shells than for heat flow distributed over all nanotube shells. The main difficulty is to quantitatively evaluate the magnitude of this difference. For this, a theoretical calculation is mandatory and that is why Ref. 13 could not conclusively settle this issue. In what follows, we give quantitative evidence of few-shell conduction, rather than homogeneous all-shell conduction, in the BNNT thermal conductivity measurements of Ref. 13. In turn, our results also suggest that in principle, thermal conductivities ten times larger than previously reported could be achieved in BNNTs, if all shells were able to contribute to the heat flow.

\section{METHODS}

Although the character and the magnitude of isotope effects on thermal conduction in bulk materials are well known, this is not the case for a complex structure such as a NT. Unlike bulk materials, in which the scattering rate is well described by the Rayleigh law, the phonon-dispersion curves of NTs are very complex, and it is not possible to predict the magnitude of isotope scattering using any sort of a Rayleigh-type expression. An atomistic description of the system is needed, along with an accurate computation of interatomic interactions. We have evaluated the effect of isotope disorder on heat flow in BNNTs using the $a b$ initio atomistic Green's function approach. ${ }^{19,20}$ The Green's functions treat isotope scattering exactly and are therefore much more accurate than any finite order calculation such as the Born approximation. They include the effect of multiple scattering, which yields quantitative differences with respect to an independent scatterer model, as we will show. To eliminate the dependence on one particular isotope configuration, the results should be averaged over an ensemble of NTs $(\sim 100)$ of the same length $L$ with a random distribution of ${ }^{10} \mathrm{~B}$ isotopes of a fixed concentration. We have performed calculations for such NTs with $20 \%{ }^{10} \mathrm{~B}$ by linking them to semi-infinite isotopically pure NT leads $\left(100 \%{ }^{11} \mathrm{~B}\right)$, which may generate artificial thermal resistance at the interfaces. 
This effect is, however, minor for $L \sim 1 \mu \mathrm{m}$, since very similar thermal conductivities (less than $3 \%$ of the difference) were obtained from the calculations where the boron mass in the leads was set to 10.8 (which corresponds to $20 \%$ ${ }^{10} \mathrm{~B}$ and, hence, the minimal mismatch with the impure region) and 10 (the maximal mismatch).

The force constants used to compute the Green's functions were obtained via an $a b$ initio calculation, i.e., without any adjustable parameters. This is advantageous because it ensures that the results do not depend on the particular parametrization employed, as would be the case if empirical potentials were used. We use a standard density-functional approach to obtain the force constants. ${ }^{21,22}$ The calculations were done in the local-density approximation, using a double zeta polarized basis set. A symmetrization procedure was subsequently applied to ensure fulfillment of translational and rotational symmetries. ${ }^{20,23}$ Details are given elsewhere. ${ }^{23}$

\section{RESULTS AND DISCUSSION}

We have calculated the thermal conductivity through an isotopically enriched single-walled $(8,0)$ BNNT $\left(100 \%{ }^{11} \mathrm{~B}\right)$ and one that contains the natural isotope concentration of $\mathrm{B}$ $\left(80 \%{ }^{11} \mathrm{~B}\right.$ and $\left.20 \%{ }^{10} \mathrm{~B}\right)$. The thermal conductivity $\kappa$ is defined as $\kappa=\sigma L / S$, where $\sigma$ is the thermal conductance, $L$ is the NT length, and $S$ is the portion of the NT cross section that contributes to heat conduction. ${ }^{24}$ NT lengths were taken to be $3 \mu \mathrm{m}$, which are the same as the experimentally investigated ones. ${ }^{25}$ The cross section of the calculated singlewalled NTs (SWNTs) was taken as $S=2 \pi R \delta,{ }^{26}$ where $R$ is the NT radius, and $\delta=3.35 \AA$ is the layer separation in bulk hexagonal $\mathrm{BN} .{ }^{27}$ The thermal conductivity of the measured multiwalled NTs (MWNTs) was estimated by incorporating the geometrical area between the inner and the outer radii as the actual cross section. ${ }^{13}$ The computational burden of the calculation prevents us from analyzing the MWNT with a diameter of $\approx 30 \mathrm{~nm}$ considered in the experiment. ${ }^{13}$ However, our results for SWNTs should be comparable to the experimental ones since theoretical calculations suggest that the thermal conductivity above $150 \mathrm{~K}$ should be very similar for SWNTs and MWNTs. ${ }^{26}$

The calculated ratio between the thermal conductivity of the isotopically pure and the $20 \%$ impure cases agrees well in the order of magnitude with the ones experimentally observed (see Table I). However, as mentioned in Sec. I, the theoretical harmonic thermal conductivities $\kappa$ for both natural and isotopically pure SWNTs at the room temperature are 1 order of magnitude larger than those reported for MWNTs. ${ }^{13}$ To understand the origin of the reduced conductivity, let us examine the two possible mechanisms mentioned earlier. We first analyze the case of an all-shell homogeneous heat flow, where the additional resistance is due to intershell scattering distributed all along the nanotube. This scattering mechanism will act in parallel with isotope scattering in impure NTs, leading to an effective mean-free path $l_{e}(\omega)$ given by Matthiessen's rule $l_{e}(\omega)^{-1}=l_{i}(\omega)^{-1}+l_{\text {is }}(\omega)^{-1}$, where $l_{i}(\omega)\left[l_{\text {is }}(\omega)\right]$ is the mean-free path due to isotope (intershell) scattering only. For pure NTs, intershell scattering limits the heat flow and $l_{e}(\omega)=l_{\text {is }}(\omega)$. In both cases, the trans-
TABLE I. The thermal conductivity ratio $\kappa_{\text {pure }} / \kappa_{\text {natural }}$ of isotopically pure versus natural nanotube thermal conductivity for several values of temperature and different scattering mechanisms taken into account.

\begin{tabular}{ccccc}
\hline \hline $\begin{array}{c}T \\
(\mathrm{~K})\end{array}$ & Ratio $^{\mathrm{a}}$ & Ratio $^{\mathrm{b}}$ & Ratio $^{\mathrm{c}}$ & Ratio $^{\mathrm{d}}$ \\
\hline 100 & 1.44 & 1.40 & 1.20 & 1.8 \\
200 & 2.13 & 1.85 & 1.50 & 1.5 \\
300 & 2.77 & 2.06 & 1.68 & 1.7 \\
\hline \hline
\end{tabular}

asotope scattering only.

${ }^{\mathrm{b}}$ Isotope + anharmonic scattering.

${ }^{\mathrm{c}}$ Isotope + anharmonic + some minor intershell scattering.

${ }^{\mathrm{d}}$ Experimental data from Ref. 13 ; estimated dispersion $\sim \pm 0.1$.

mission can be given approximately as $\mathcal{T}(\omega)=N(\omega) /[1$ $\left.+L / l_{e}(\omega)\right]$, where $N(\omega)$ is the number of phonon bands at frequency $\omega$ for the isotopically pure NT (shown in the inset of Fig. 1). The extraction of the mean-free paths for isotope scattering in BNNTs (see Fig. 1) was done from our harmonic calculation, by fitting the exact results for the transmission to the expression $N(\omega) /\left[1+L / l_{i}(\omega)\right]$ for a range of lengths $L .{ }^{16}$ The mean-free path due to intershell scattering may depend on frequency, but it does not depend on temperature, since it is a harmonic process. The specific dependence will be determined by the relative orientation of the shells and the related stresses and dislocations that might build up. Rather than attacking this extremely intricate issue, it is reasonable to try various analytical dependences of the type $l_{\text {is }}^{-1} \sim \omega^{n}$ and evaluate the result for different values of $n$. In all cases (with $n$ ranging from 0 to 4 ), the calculated $\kappa$ could be reduced by an order of magnitude to match the experiment only if the intershell scattering mean-free path was much shorter than the one due to isotope scattering in most of the frequency spectrum. Consequently, the effect of isotope scattering would be virtually unobservable, which clearly cannot be the case, as seen in the experiment. As an example, we were able to fit the measured $\kappa_{\text {pure }}$

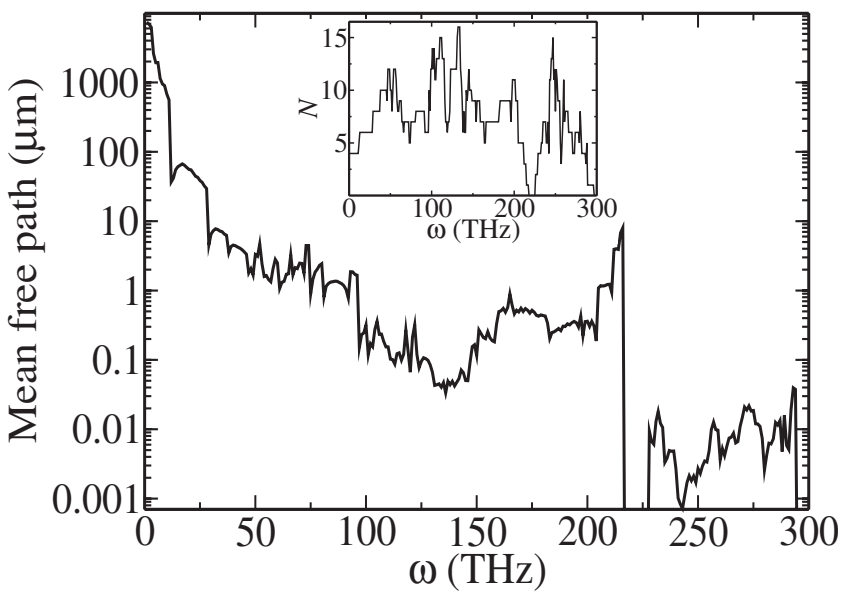

FIG. 1. Isotope scattering mean-free path versus frequency for natural $\left(80 \%{ }^{11} \mathrm{~B}\right.$ and $\left.20 \%{ }^{10} \mathrm{~B}\right)(8,0)$ boron nitride nanotubes. Inset: The number of phonon bands for the pure $\left(100 \%{ }^{11} \mathrm{~B}\right)$ nanotube as a function of frequency. 


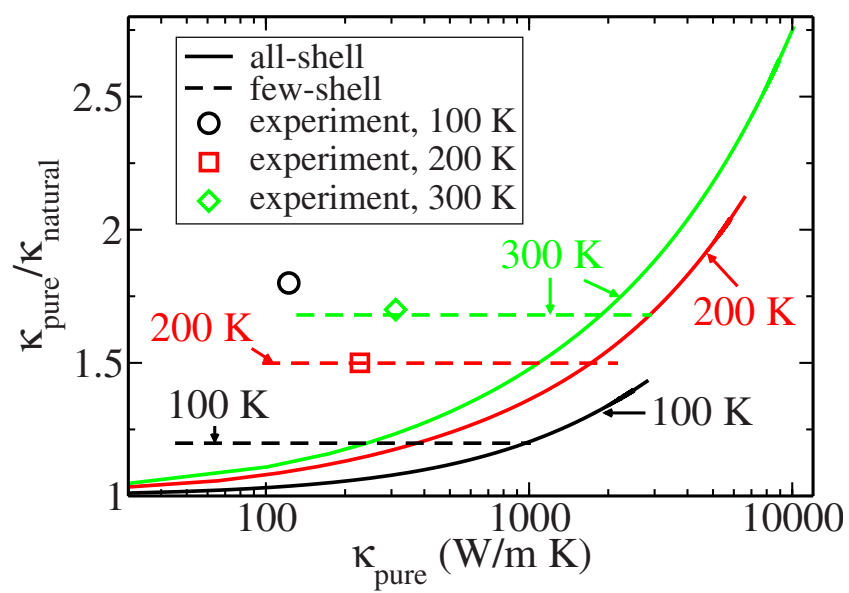

FIG. 2. (Color online) Thermal conductivity ratio of isotopically pure $\left(100 \%{ }^{11} \mathrm{~B}\right)$ and natural $\left(80 \%{ }^{11} \mathrm{~B}\right.$ and $\left.20 \%{ }^{10} \mathrm{~B}\right)(8,0)$ boron nitride nanotubes as a function of thermal conductivity of the isotopically pure nanotube for several temperatures. Solid black (100 $\mathrm{K})$, red (dark gray) (200 K), and green (light gray) (300 K) lines: all-shell conduction case where the intershell scattering mean-free path is varied (see the text). Dashed black $(100 \mathrm{~K})$, red (dark gray), $(200 \mathrm{~K})$ and green (light gray) $(300 \mathrm{~K})$ lines: few-shell conduction case where the number of effective active shells is varied (see the text), with the anharmonic and the intershell scattering lengths adjusted to reproduce the experimental results from Ref. 13 given by the circle $(100 \mathrm{~K})$, the square $(200 \mathrm{~K})$, and the diamond $(300 \mathrm{~K})$.

$\approx 311 \mathrm{~W} / \mathrm{m} \mathrm{K}$ at $300 \mathrm{~K}$ with $l_{\text {is }}(\omega)=9.2 \mathrm{~nm}(n=0)$. However, when we incorporated the effect of isotope scattering using $l_{i}(\omega)$ given in Fig. $1, \kappa_{\text {natural }}$ was only slightly reduced to $298 \mathrm{~W} / \mathrm{m} \mathrm{K}$, which clearly overestimates the measured value of $\approx 186 \mathrm{~W} / \mathrm{m} \mathrm{K}$. This is further illustrated in Fig. 2, which shows that $\kappa_{\text {pure }}$ obtained by varying the mean-free path due to intershell scattering for $n=0$ [solid lines: black $(100 \mathrm{~K})$, red/dark gray $(200 \mathrm{~K})$, and green/light gray (300 $\mathrm{K})$ ], agrees with the experimental values only if $\kappa_{\text {pure }} / \kappa_{\text {natural }}$ becomes close to 1 . We therefore discard the possibility that intershell scattering is a determinant as speculated in the experimental study. For the same reason, any other scattering mechanism such as anharmonicity cannot lead to such a large decrease in $\kappa$ without masking the effect of isotope scattering.

We next discuss whether the low thermal conductivities reported in the experiment are due to contact resistance. Abrupt junctions between suspended NTs and contacts lead to significant contact resistance due to the large acoustic mismatch, whereas extended adiabatic contacting minimizes this effect. In order to deduce the importance of contacts in the BNNT measurements, we use the same argument as for intershell scattering. A contact contributes an additive term to the inverse of the transmission function and, similarly as shown for the case of intershell scattering, it would diminish the experimentally observed isotope effect if its influence was appreciable.

Next we focus on the second possibility: inefficient heat transfer from the MWNT outermost shell to the inner shells. In Ref. 13, heat flow is assumed in all MWNT layers and the cross section used to determine the thermal conductivity is $\pi\left(d_{2}^{2}-d_{1}^{2}\right) / 4$, where $d_{1}=8.8 \mathrm{~nm}$ and $d_{2}=32 \mathrm{~nm}$ are the inner and outer diameters, respectively. ${ }^{25}$ However, if heat is carried by the outermost shell only, the effective cross section is $\pi\left(d_{2}-2 \delta\right) \delta$. The ratio of the MWNT cross section to the outermost shell cross section $(\approx 22.5)$ provides an upper limit to the rescaling factor that needs to be introduced in the experimentally reported $\kappa$ to account for inefficient heat transfer in MWNTs. The heat conduction will be confined to the outer shells for both natural and isotopically enriched BN MWNTs and the measured thermal conductivity can be rescaled by the same factor for MWNTs with similar diameters. Consequently, in contrast to the all-shell conduction case shown by solid lines in Fig. $2, \kappa_{\text {pure }} / \kappa_{\text {natural }}$ is independent of $\kappa_{\text {pure }}$ if only a few shells conduct. If we rescale the calculated $\kappa$ with the factor of 22.5 , we indeed obtain a fairly good agreement between the predicted and the measured $\kappa$ for natural abundance BNNTs in much of the temperature range [given by dashed red/gray lines and squares in Fig. $3(\mathrm{a})$, respectively]. This implies a rather strong confinement of propagating phonons in a few outermost shells.

We should note that a more detailed picture of few-shell conduction versus intershell scattering in the MWNT thermal transport could be obtained by analyzing double-walled or triple-walled incommensurate NTs with small diameters. This would require extremely demanding computations on very large supercells. In commensurate systems, interlayer coupling modifies the phonon dispersions, ${ }^{28}$ but it does not introduce an additional scattering mechanism. Only incommensurate layers produce scattering. Although such detailed studies are possible, ${ }^{29-32}$ they would not lead to any different conclusions than the ones presented here: heat conduction in the measured BN MWNTs takes place through a few shells only. Any hypothetical type of scattering acting along the NTs capable of reducing the thermal conductivity $\sim 10$ times with respect to the ballistic limit would completely mask the isotope effect and therefore contradict the experimental evidence. $^{13}$

Even when inefficient heat transfer into the inner layers is taken into account, the calculation overestimates the measured $\kappa$ of isotopically pure BNNTs [compare dotted black line and circles in Fig. 3(a), respectively]. The discrepancy increases with temperature and it is due to anharmonic scattering. In a rough approximation, anharmonic effects can be taken into account by introducing an anharmonic relaxation length $l_{\phi}$ as a contribution to the effective transport length in Matthiessen's rule. In order to estimate its value, we use an expression given by Klemens and Pedraza: ${ }^{33} l_{\phi}=A T^{-1} \omega^{-2}$.

By fitting the calculated $\kappa_{\text {pure }}$ to the experimental one [compare green/light gray line and circles in Fig. 3(a)], we obtain $A=1.5 \times 10^{25} \mathrm{~m} \mathrm{~K} \mathrm{~s}^{-2}$ in the case of BN. We find a similar factor when comparing our theoretical results for CNTs with the measured ones. ${ }^{11,12}$ This finding contrasts the experimental reports that the thermal conductivity of bulk $\mathrm{BN}$ is several times smaller than that of graphite. ${ }^{34}$ However, Ref. 34 shows that the limiting mechanism to the phonon flow in bulk hexagonal $\mathrm{BN}$ is due to dislocations. This can explain why the thermal conductivity of bulk BN is smaller than that of graphite, in spite of similar phonon dispersions. Furthermore, absence of dislocations in BN MWNTs, in conjunction to the outermost shell conduction, can explain similar thermal conductivities of $\mathrm{C}$ and BN MWNTs. If this is the 


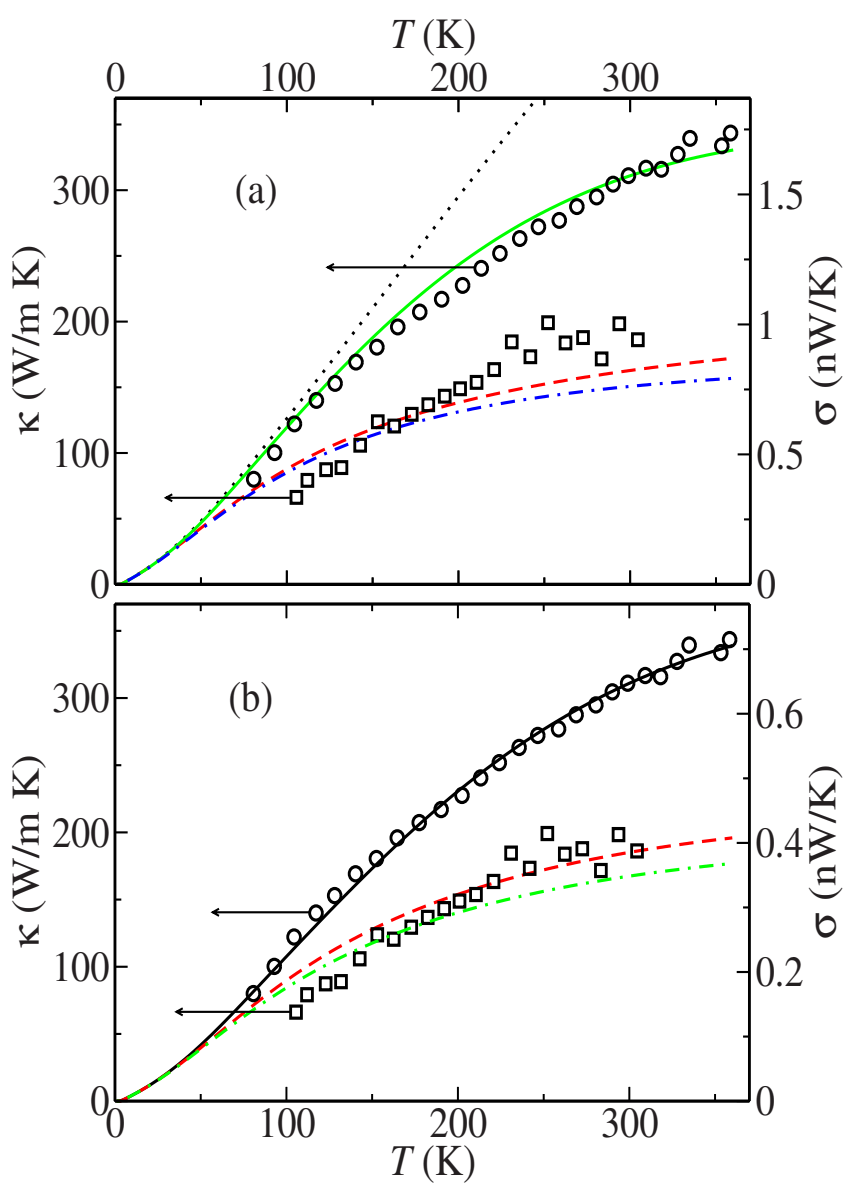

FIG. 3. (Color online) Thermal conductivity $\kappa$ and thermal conductance $\sigma$ as a function of temperature for $(8,0)$ boron nitride nanotubes, showing the effect of different scattering mechanisms. All calculated thermal-conductivity results are rescaled according to the few-shell model with (a) 1 and (b) 2.5 effective active shells (see the text), for comparison with the experimental curves for multiwalled nanotubes. Black circles and squares denote the experimental thermal conductivities for the isotopically pure $\left(100 \%{ }^{11} \mathrm{~B}\right)$ and natural $\left(80 \%{ }^{11} \mathrm{~B}\right.$ and $\left.20 \%{ }^{10} \mathrm{~B}\right)$ nanotubes from Ref. 13 , respectively. (a) Isotope scattering only: dotted black (pure) and dashed red/gray (natural) lines. Isotope and anharmonic scattering: solid green/light gray (pure) and dash-dotted blue/dark gray (natural) lines. (b) Isotope, anharmonic, and intershell scattering: solid black (pure) and dashed red/gray (natural) lines. The effect of neglecting wave interference for the natural case is given by dashdotted green/light gray curve.

case, anharmonic scattering should be the limiting factor for thermal conduction in MWNTs and it should be similar for CNTs and BNNTs.

Although the empirical inclusion of anharmonic scattering gives the correct shape of the calculated curve in the isotopically pure case, the $\kappa_{\text {pure }} / \kappa_{\text {natural }}$ ratio does not correspond to the experimental one for higher temperatures [see column $b$ in Table I and dash-dotted blue/dark gray line against squares in Fig. 3(b) ]. This points out the influence of additional harmonic scattering mechanisms, such as intershell scattering. One can model the contribution of these effects by a constant contribution to the effective mean-free path. The relatively long value of $1.7 \mu \mathrm{m}$, in conjunction with the anharmonic and the rescaling factors of $A=1 \times 10^{25} \mathrm{~m} \mathrm{~K} \mathrm{~s}^{-2}$ and 9.4, respectively, gives a good agreement of the calculated curves with the measured ones in most of the temperature range [compare solid black line with circles in Fig. 3(b) for the isotopically pure NT and dashed red/gray line with squares for the natural abundance one]. We also show in dashed lines in Fig. 2 the dependence of $\kappa_{\text {pure }} / \kappa_{\text {natural }}$ on $\kappa_{\text {pure }}$, which is obtained by varying the rescaling factor from 1 to 22.5, while anharmonic and intershell scattering are kept constant. The actual rescaling factor of 9.4 is below the upper limit of 22.5 and confirms that heat flow is restricted to two to three outermost shells effectively. If one could improve heat transfer to the inner shells (perhaps by using longer contacts), so that all shells contributed to the heat flow, it would be possible to achieve 1 order of magnitude larger $\kappa$. At low temperatures, thermal conductivities of SWNTs and MWNTs become appreciably different due to the three-dimensional (3D) character of the latter and the quasi-one-dimensional (quasi-1D) character of the former. ${ }^{26}$ The coupling between the planes in bulk hexagonal BN induces the $T^{2.5}$ dependence of the thermal conductivity at low $T,{ }^{26}$ in contrast to the linear $T$ dependence in SWNTs. Since the effect of this coupling on the heat conduction in thick MWNTs is similar to that of hexagonal BN (except at extremely low temperatures), ${ }^{26}$ one cannot expect a good agreement between the calculated and the measured curves for the temperatures below $150 \mathrm{~K}$.

In Fig. 3(b) we also show in dash-dotted green/light gray line $\kappa_{\text {natural }}$ if wave interference effects due to scattering from isotopes are neglected. This is done by assuming that all scattering events are independent. ${ }^{35}$ The transmission of a single-scattering event $\mathcal{T}_{1}$ is calculated in the NT with one isotope only and the total transmission is given as $1 / \mathcal{T}$ $=N_{i} / \mathcal{T}_{1}-\left(N_{i}-1\right) / \mathcal{T}_{0}$, where $N_{i}$ is the number of isotopes, and $\mathcal{T}_{0}$ is the transmission through the pure NT. Comparison with the curve including wave interference effects shows a fairly good estimation of the thermal conductivity even with this simplified approach.

\section{CONCLUSION}

In the light of the atomistic calculations presented, we conclude that heat conduction in previous experiments on BN MWNTs is confined to just a few outermost shells; whereas scattering along the nanotubes alone cannot account for the low experimental values of thermal conductivity, in contrast to earlier suggestions. The analysis implies that thermal conductivities on the order of thousands of $\mathrm{W} / \mathrm{m} \mathrm{K}$ might be achieved in BN MWNTs, and perhaps also in bulk $\mathrm{BN}$, provided that the samples are highly ordered and well contacted.

\section{ACKNOWLEDGMENTS}

We thank C. W. Chang and A. Majumdar for providing the dimensions of the NTs in Ref. 13. We are grateful to S. Roche for helpful discussions. This project was supported by the European Union under Grant No. MIRG-CT-2006039302 and by NSF under Grants No. 0651310 and No. 0651427. 
*ivana.savic@cea.fr

†natalio.mingo@cea.fr

${ }^{1}$ D. G. Cahill, W. K. Ford, K. E. Goodson, G. D. Mahan, A. Majumdar, H. J. Maris, R. Merlin, and S. R. Phillpot, J. Appl. Phys. 93, 793 (2003).

${ }^{2}$ R. Saito, G. Dresselhaus, and M. Dresselhaus, Physical Properties of Carbon Nanotubes (Imperial College Press, London, 1998).

${ }^{3}$ A. Rubio, J. L. Corkill, and M. L. Cohen, Phys. Rev. B 49, 5081(R) (1994).

${ }^{4}$ X. Blase, A. Rubio, S. G. Louie, and M. L. Cohen, Europhys. Lett. 28, 335 (1994).

${ }^{5}$ N. G. Chopra, R. J. Luyken, K. Cherrey, V. H. Crespi, M. L. Cohen, S. G. Louie, and A. Zettl, Science 269, 966 (1995).

${ }^{6}$ Z. Weng-Sieh, K. Cherrey, N. G. Chopra, X. Blase, Y. Miyamoto, A. Rubio, M. L. Cohen, S. G. Louie, A. Zettl, and R. Gronsky, Phys. Rev. B 51, 11229(R) (1995).

${ }^{7}$ P. Kim, L. Shi, A. Majumdar, and P. L. McEuen, Phys. Rev. Lett. 87, 215502 (2001).

${ }^{8}$ L. Shi, D. Li, C. Yu, W. Jang, D. Kim, Z. Yao, P. Kim, and A. Majumdar, ASME Trans. J. Heat Transfer 125, 881 (2003).

${ }^{9}$ M. Fujii, X. Zhang, H. Xie, H. Ago, K. Takahashi, T. Ikuta, H. Abe, and T. Shimizu, Phys. Rev. Lett. 95, 065502 (2005).

${ }^{10}$ H.-Y. Chiu, V. V. Deshpande, H. W. C. Postma, C. N. Lau, C. Miko, L. Forro, and M. Bockrath, Phys. Rev. Lett. 95, 226101 (2005).

${ }^{11}$ C. Yu, L. Shi, Z. Yao, D. Li, and A. Majumdar, Nano Lett. 5, 1842 (2005).

${ }^{12}$ E. Pop, D. Mann, Q. Wang, K. Goodson, and H. Dai, Nano Lett. 6, 96 (2006).

${ }^{13}$ C. W. Chang, A. M. Fennimore, A. Afanasiev, D. Okawa, T. Ikuno, H. Garcia, D. Li, A. Majumdar, and A. Zettl, Phys. Rev. Lett. 97, 085901 (2006).

${ }^{14}$ C. W. Chang, D. Okawa, A. Majumdar, and A. Zettl, Science 314, 1121 (2006).

${ }^{15}$ C. W. Chang, D. Okawa, H. Garcia, T. D. Yuzvinsky, A. Majumdar, and A. Zettl, Appl. Phys. Lett. 90, 193114 (2007).
${ }^{16}$ I. Savić, N. Mingo, and D. A. Stewart, Phys. Rev. Lett. 101, 165502 (2008)

${ }^{17}$ G. Stoltz, M. Lazzeri, and F. Mauri, arXiv:0810.1830 (unpublished).

${ }^{18}$ G. Zhang and B. Li, J. Chem. Phys. 123, 114714 (2005).

${ }^{19}$ N. Mingo and L. Yang, Phys. Rev. B 68, 245406 (2003).

${ }^{20}$ N. Mingo, D. A. Stewart, D. A. Broido, and D. Srivastava, Phys. Rev. B 77, 033418 (2008).

${ }^{21}$ J. M. Soler, E. Artacho, J. D. Gale, A. García, J. Junquera, P. Ordejón, and D. Sánchez-Portal, J. Phys.: Condens. Matter 14, 2745 (2002).

${ }^{22}$ D. Sánchez-Portal, E. Artacho, J. M. Soler, A. Rubio, and P. Ordejón, Phys. Rev. B 59, 12678 (1999).

${ }^{23}$ D. A. Stewart, I. Savić, and N. Mingo, Nano Lett. (to be published).

${ }^{24}$ S. Datta, Electronic Transport in Mesoscopic Systems (Cambridge University Press, Cambridge, England, 1995).

${ }^{25} \mathrm{C}$. W. Chang (private communication).

${ }^{26}$ N. Mingo and D. A. Broido, Phys. Rev. Lett. 95, 096105 (2005).

${ }^{27}$ A. Celik-Aktas, J.-M. Zuo, J. F. Stubbins, C. Tang, and Y. Bando, Acta Crystallogr., Sect. A: Found. Crystallogr. 61, 533 (2005).

${ }^{28}$ J. Serrano, A. Bosak, R. Arenal, M. Krisch, K. Watanabe, T. Taniguchi, H. Kanda, A. Rubio, and L. Wirtz, Phys. Rev. Lett. 98, 095503 (2007).

${ }^{29}$ S. Roche, F. Triozon, A. Rubio, and D. Mayou, Phys. Rev. B 64, 121401(R) (2001).

${ }^{30}$ F. Triozon, S. Roche, A. Rubio, and D. Mayou, Phys. Rev. B 69, 121410(R) (2004).

${ }^{31}$ S. Wang and M. Grifoni, Phys. Rev. Lett. 95, 266802 (2005).

${ }^{32}$ S. Wang, M. Grifoni, and S. Roche, Phys. Rev. B 74, 121407(R) (2006).

${ }^{33}$ P. G. Klemens and D. F. Pedraza, Carbon 32, 735 (1994).

${ }^{34}$ E. K. Sichel, R. E. Miller, M. S. Abrahams, and C. J. Buiocchi, Phys. Rev. B 13, 4607 (1976).

${ }^{35}$ T. Markussen, R. Rurali, A.-P. Jauho, and M. Brandbyge, Phys. Rev. Lett. 99, 076803 (2007). 